\title{
TSPO Versus P2X7 as a Target for Neuroinflammation: An In Vitro and In Vivo Study
}

Donatienne Van Weehaeghe ${ }^{1}$, Evelien Van Schoor ${ }^{2-4}$, Joke De Vocht ${ }^{2}$, Michel Koole $^{1}$, Bala Attili5 ${ }^{5}$, Sofie Celen ${ }^{5}$, Lieven Declercq ${ }^{5}$, Dietmar R. Thal ${ }^{3,4,6}$, Philip Van Damme ${ }^{2}$, Guy Bormans ${ }^{5}$, and Koen Van Laere ${ }^{1}$

${ }^{I}$ Division of Nuclear Medicine, Department of Imaging and Pathology, University Hospitals Leuven, KU Leuven, Leuven, Belgium; ${ }^{2}$ Department of Neurology, University Hospitals Leuven, and Laboratory of Neurobiology, Center for Brain and Disease Research, VIB, KU Leuven, Leuven, Belgium; ${ }^{3}$ Leuven Brain Institute, KU Leuven, Leuven, Belgium; ${ }^{4}$ Laboratory for Neuropathology, Department of Imaging and Pathology, KU Leuven, Leuven, Belgium; ${ }^{5}$ Laboratory for Radiopharmaceutical Research, Department of Pharmaceutical and Pharmacological Sciences, KU Leuven, Leuven, Belgium; and ${ }^{6}$ Department of Pathology, UZ Leuven, Leuven, Belgium

Neuroinflammation is important in amyotrophic lateral sclerosis (ALS). The P2X7 receptor (P2X7R) is a promising target for neuroinflammation. The objective of this study was to compare ${ }^{18} \mathrm{~F}-\mathrm{DPA} 714$, a second-generation translocator protein tracer, with ${ }^{11} \mathrm{C}-J N J 717$, a novel P2X7R tracer, in vitro and in vivo in ALS. Methods: For the in vitro portion of the study, autoradiography with ${ }^{18} \mathrm{~F}-\mathrm{DPA} 714$ and ${ }^{11} \mathrm{C}-$ JNJ717 was performed on human ALS brain sections in comparison to immunofluorescence with Iba1 and GFAP. For the in vivo portion, 3 male patients with early-stage ALS $(59.3 \pm 7.2$ y old) and 6 healthy volunteers ( $48.2 \pm 16.5$ y old, 2 men and 4 women) underwent dynamic PET/MR scanning with ${ }^{18} \mathrm{~F}-\mathrm{DPA} 714$ and ${ }^{11} \mathrm{C}-J \mathrm{NJ} 717$. Volumeof-distribution images were calculated using Logan plots and analyzed on a volume-of-interest basis. Results: Autoradiography showed no difference in ${ }^{11} \mathrm{C}-J N J 717$ binding but did show increased ${ }^{18} \mathrm{~F}$-DPA714 binding in the motor cortex correlating with lba1 expression (glial cells). Similar findings were observed in vivo, with a $13 \%$ increase in ${ }^{18} \mathrm{~F}$ DPA714 binding in the motor cortex. Conclusion: In symptomatic ALS patients, ${ }^{18} \mathrm{~F}$-DPA714 showed increased signal whereas ${ }^{11} \mathrm{C}$ JNJ717 was not elevated.

Key Words: translocator protein (TSPO); P2X7; neuroinflammation; amyotrophic lateral sclerosis; brain specimen

J Nucl Med 2020; 61:604-607

DOI: 10.2967/jnumed.119.231985

G

lial activation is important in amyotrophic lateral sclerosis (ALS) (1-3). Measurement may be of value for patient selection and assessment of therapy effectiveness. Until now, the most widely used PET target to study glial activation has been the translocator protein (TSPO). Several ALS studies demonstrated increased glial activation in motor and premotor regions, temporal cortex, pons, thalamus, and corticospinal tract (4-6). However, TSPO imaging has some drawbacks: relatively low signal-to-noise ratio, endothelial binding (7), and the need for genotyping before scanning (8). Therefore, other neuroinflammation targets have

Received Jun. 3, 2019; revision accepted Aug. 26, 2019.

For correspondence or reprints contact: Donatienne Van Weehaeghe, UZ Leuven, Herestraat 49, Leuven, 3000 Belgium.

E-mail: donatienne.vanweehaeghe@kuleuven.be

Published online Sep. 27, 2019.

COPYRIGHT (C 2020 by the Society of Nuclear Medicine and Molecular Imaging. been investigated, such as the membrane $\mathrm{P} 2 \mathrm{X} 7$ receptor $(\mathrm{P} 2 \mathrm{X} 7 \mathrm{R})$. Activation of this receptor induces NLPR3 inflammasome formation and releases proinflammatory cytokines and reactive oxygen species. Recently, we have developed ${ }^{11} \mathrm{C}-J N J 717$, a P2X7R radioligand with a nanomolar affinity $(1.6 \mathrm{nM})$, high specificity, and blood-brain barrier penetration. The tracer was validated in an animal model of neuroinflammation (9) and had a good test-retest variability in humans $(10 \%-15 \%)(10)$.

The aim of this study was to perform a head-to-head comparison between the second-generation TSPO tracer ${ }^{18} \mathrm{~F}$-labeled DPA714 ( $N, N$-diethyl-2-[4-(2-fluoroethoxy)phenyl]-5,7-dimethylpyrazolo[1,5a]pyrimidine-3-acetamide) and ${ }^{11} \mathrm{C}$-labeled JNJ717 ([ $\left.\left.{ }^{11} \mathrm{C}\right] J N J 54173717\right)$ in vitro using brain slices of ALS patients and in vivo in ALS patients and healthy volunteers (HVs).

\section{MATERIALS AND METHODS}

\section{In Vitro}

Snap-frozen $5-\mu \mathrm{m}$ brain slices of 2 male ALS patients (mean age \pm $\mathrm{SD}, 61.0 \pm 10.0 \mathrm{y})$ were obtained using a cryotome. The occipital cortex (OC) was used as a control region because neuroinflammation in ALS is extensive in the motor cortex (MC) but negligible in the OC

TABLE 1

Subject Demographics

\begin{tabular}{lccccccc}
\hline $\begin{array}{c}\text { Subject } \\
\text { no. }\end{array}$ & Group & $\begin{array}{c}\text { Age } \\
(\mathrm{y})\end{array}$ & $\begin{array}{c}\text { Weight } \\
(\mathrm{kg})\end{array}$ & $\begin{array}{c}\text { Dose } \\
(\mathrm{MBq})\end{array}$ & Sex & ALSFRS-r & FVC \\
\hline 1 & HV & 25 & 56 & 163 & $\mathrm{~F}$ & - & - \\
2 & $\mathrm{HV}$ & 37 & 73 & 131 & $\mathrm{M}$ & - & - \\
3 & $\mathrm{HV}$ & 39 & 65 & 80 & $\mathrm{~F}$ & - & - \\
4 & $\mathrm{HV}$ & 60 & 66 & 144 & $\mathrm{~F}$ & - & - \\
5 & HV & 53 & 78 & 152 & $\mathrm{~F}$ & - & - \\
6 & HV & 75 & 70 & 163 & $\mathrm{M}$ & - & - \\
7 & ALS & 59 & 84 & 149 & $\mathrm{M}$ & 43 & 113 \\
\hline 8 & ALS & 50 & 68 & 161 & $\mathrm{M}$ & 39 & 86 \\
9 & ALS & 68 & 63 & 134 & $\mathrm{M}$ & 45 & 126 \\
\hline
\end{tabular}

ALSFRS-r $=$ revised ALS functional rating scale; FVC = functional vital capacity. 
$(4,6,11)$. Autoradiography was performed according to the protocol described by Ory et al. (9). Blocking percentages were calculated as (digital light units $/ \mathrm{mm}^{2}$ in the presence of $20 \mu \mathrm{M}$ blocker)/(digital light units $/ \mathrm{mm}^{2}$ tracer only).

For immunohistochemistry, astrocytes and microglia were labeled with polyclonal rabbit anti-glial fibrillary acidic protein (GFAP) (1:300, Z0334, Dako; Agilent) and monoclonal mouse anti-ionized calcium binding adaptor molecule 1 (Iba1) (1:300, GT10312; Genetex). Afterward, the sections were incubated with goat antirabbit Cy5 (1:100, 111-175-144; Jackson Immunoresearch) and goat antimouse Cy3 (1:100, 115-165-003; Jackson Immunoresearch). The slides were mounted with ProLong Gold Antifade Mountant (Thermo Fisher Scientific). Wholesection images were acquired with a NanoZoomer S60 digital slide scanner (Hamamatsu Photonics) at $\times 20$ magnification. The number of Iba1-positive and GFAP-positive cells was quantified per square millimeter in the area with highest density.

\section{In Vivo}

Six HVs (mean, $48.2 \pm 16.5$ y old; 2 men and 4 women, all mediumaffinity binders) and 3 male ALS patients (59.3 \pm 7.2 y old; 2 mediumaffinity binders and 1 high-affinity binder) were included (Table 1). All subjects were genotyped for the rs6971 polymorphism and the 13 P2X7R exons (Supplemental Table 1; supplemental materials are available at http://jnm.snmjournals.org). The main exclusion criteria for HVs were low-affinity binding, abnormal findings on physical/neurologic/ paraclinical examination, severe medical illness, and use of nonsteroidal antiphlogistics or corticosteroids (except for inhalation corticosteroids). ALS was diagnosed as probable or definite according to the AwajiShima or El Escorial criteria by an expert in neuromuscular disorders. The ALS patients had sporadic ALS disease and had been symptomatic for less than $1 \mathrm{y}$.

The study was performed in accordance with the World Medical Association Declaration of Helsinki and approved by the local University Ethics Committee. Written informed consent was obtained from all subjects before study participation.

Data were acquired on a Signa 3-T simultaneous PET/MR system (GE Healthcare) in list mode. All subjects underwent a 60-min ${ }^{18}$ F-DPA714 scan $(251 \pm 44 \mathrm{MBq})$ and a 70-min ${ }^{11}$ C-JNJ717 scan (142 \pm 44 MBq). Multichannel segmentation (SPM, version 12) was performed to calculate subject-specific tissue probability maps (gray matter, white matter, and cerebrospinal fluid) using 2 imaging sequences of GE Healthcare: Bravo (a 3-dimensional volumetric T1-weighted sequence in the oblique plane, with an echo time of $3.2 \mathrm{~ms}$, a repetition time of $8.5 \mathrm{~ms}$, an inversion time of $450 \mathrm{~ms}$, a flip angle of 12, a receiver bandwidth of 31.2 , and 1 excitation) and Cube FLAIR (a 3-dimensional T2-weighted, fluid-attenuated inversion recovery sequence in the oblique plane, with an echo time of $136 \mathrm{~ms}, 1$ echo, an echo train length of 190, a repetition time of $8,500 \mathrm{~ms}$, an inversion time of $50 \mathrm{~ms}$, a receiver bandwidth of 31.25 , and 1 excitation).

PET data were reconstructed with orderedsubsets expectation maximization (28 subsets and 4 iterations) including time-of-flight information, resolution modeling, and in-plane gaussian postreconstruction smoothing (4 mm in full width half maximum). Every frame was rigidly coregistered to the averaged first 10 frames to correct for motion.

Arterial blood samples were taken from 0 (time of tracer injection) until the end of the scan. Radiometabolites in the plasma were analyzed to determine the percentage of intact tracer. Three exponential model curves were fitted to blood and plasma activity, whereas plasma radiometabolite data were fitted with a sigmoid function for ${ }^{11} \mathrm{C}-\mathrm{JNJ} 717$ and a Hill function for ${ }^{18} \mathrm{~F}$-DPA714.

Spatial normalization (Montreal Neurological Institute template) was performed using both Bravo and 3-tissue-probability-map transformation (PMOD, version 3.7; PMOD Technologies). Voxel-based $\mathrm{V}_{\mathrm{T}}$ images were created using Logan graphical analysis (PMOD, version 3.7) with blood volume fixed to $5 \%$ and
FIGURE 1. In vitro autoradiography experiments on MC and OC slices of 2 ALS patients. (A) Sections incubated with ${ }^{18} \mathrm{~F}$-DPA714. (B) Sections incubated with ${ }^{18} \mathrm{~F}$-DPA714 in presence of PK11195. (C) Sections incubated with ${ }^{11} \mathrm{C}-J N J 717$. (D) Sections incubated with ${ }^{11} \mathrm{C}-J N J 717$ in presence of A740003. (E) Immunohistochemistry results using lba1 and GFAP. Scale bars represent $2.5 \mathrm{~mm}$. $\mathrm{WM}=$ white matter; $\mathrm{GM}$ = gray matter. 
$\mathrm{t}^{*}$ (equilibration time from which the plot is considered linear) to 31 and $36 \mathrm{~min}$ for ${ }^{18} \mathrm{~F}-\mathrm{DPA} 714$ and ${ }^{11} \mathrm{C}$-JNJ717, respectively.

Region-based partial-volume correction (FreeSurfer, version 6.0; Laboratory for Computational Neuroimaging) was applied, and the atlas of Desikan et al. was used to delineate regions (12). After partialvolume correction, the ratio $\mathrm{MC}$ over $\mathrm{OC}$ was calculated to correct for genotype differences: Ratio $=\left[\right.$ average mean $\mathrm{V}_{\mathrm{T}}$ value in precentral and paracentral gray matter bilaterally]/[average mean $V_{T}$ value in occipital gray matter bilaterally].

Nonparametric Wilcoxon-rank tests were performed in SPSS (version 25.0; IBM).

\section{RESULTS}

\section{In Vitro}

Autoradiography showed increased ${ }^{18} \mathrm{~F}$-DPA714 uptake in the MC compared with the OC (ratio, 1.7) and full blocking (99.9\%) (Fig. 1). No difference was observed in ${ }^{11} \mathrm{C}-\mathrm{JNJ} 717$ uptake between the 2 cortices with intermediate blocking (range, 58\%-83\%) with A740003, indicating a significant degree of nonspecific or off-target binding.

Immunohistochemistry showed a 4-fold higher number of microglia (Iba1-positive cells) in the MC $\left(69 / \mathrm{mm}^{2}\right)$ than in the OC $\left(17.5 / \mathrm{mm}^{2}\right)$. In contrast, only a small increase in astrocytes (GFAP-positive cells) was observed in the MC $\left(72 / \mathrm{mm}^{2}\right)$, compared with the OC $\left(57 / \mathrm{mm}^{2}\right)$.

We observed regional colocalization between Iba1 staining and ${ }^{18}$ F-DPA714 uptake. No apparent colocalization between ${ }^{18} \mathrm{~F}$ DPA714 and GFAP staining or between ${ }^{11} \mathrm{C}-J N J 717$ and Iba1/GFAP staining was detected visually.

\section{In Vivo}

In all ALS patients, we observed an increase in MC uptake for ${ }^{18} \mathrm{~F}-\mathrm{DPA} 714$ but not for ${ }^{11} \mathrm{C}-\mathrm{JNJ} 717$ (Fig. 2). In patient 3, who presented with frontotemporal dysfunction, there was also an increase in frontal cortex uptake for ${ }^{18} \mathrm{~F}-\mathrm{DPA} 714$. The ratio MC over OC without partial-volume correction was $1.03 \pm 0.04$ for ALS and $0.98 \pm 0.04$ for HV using ${ }^{18} \mathrm{~F}$-DPA714 $(P=0.20)$ and $1.00 \pm$ 0.06 for ALS and $0.97 \pm 0.04$ for $\mathrm{HV}$ using ${ }^{11} \mathrm{C}-\mathrm{JNJ} 717(P=0.61)$.

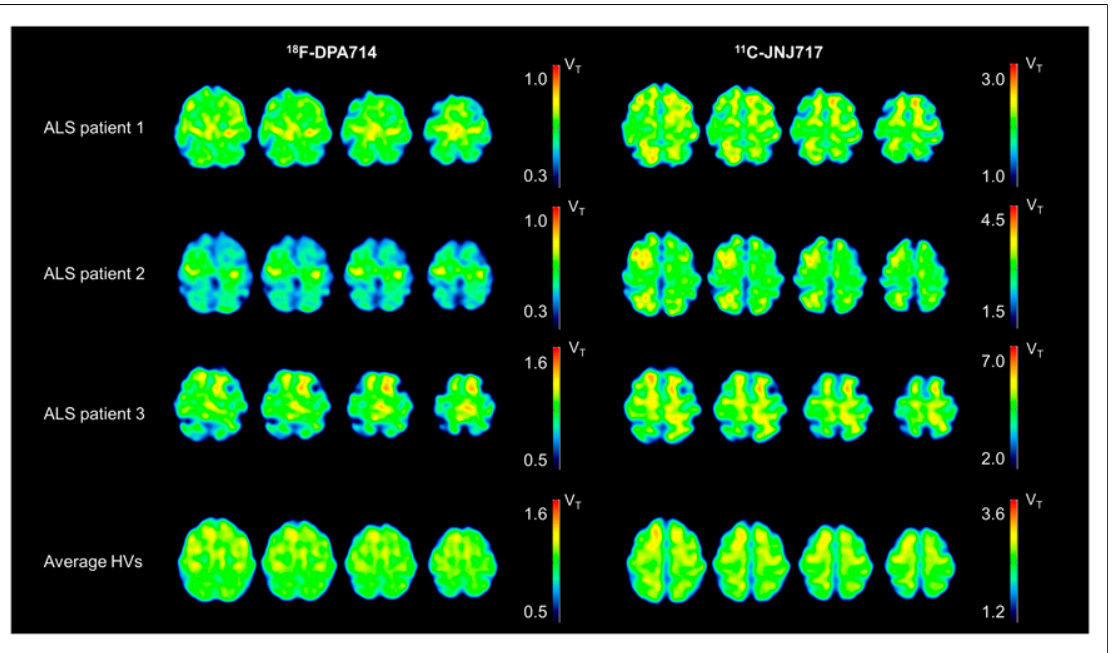

FIGURE 2. Overview of $\mathrm{V}_{\mathrm{T}}$ images $\left({ }^{18} \mathrm{~F}-\mathrm{DPA} 714\right.$ and $\left.{ }^{11} \mathrm{C}-\mathrm{JNJ} 717\right)$ of all ALS patients and average image of all HVs. Because of genotype effects for both tracers, different $V_{T}$ thresholds have been used. ALS patient 1 is 59 -y-old man, ALS patient 2 is 50 -y-old man, and ALS patient 3 is 68 $y$-old man (the patient with associated frontotemporal dysfunction).
After partial-volume correction, the ratio was $1.10 \pm 0.05$ for ALS and $0.97 \pm 0.08$ for $\mathrm{HV}$ using ${ }^{18} \mathrm{~F}-\mathrm{DPA} 714(P=0.07)$ and $1.07 \pm$ 0.09 for ALS and $1.04 \pm 0.07$ for HV using ${ }^{11} \mathrm{C}-\mathrm{JNJ} 717(P=0.80)$. In this study, none of the subjects had the previously described P2X7R polymorphism (rs3751143) that may affect $\mathrm{V}_{\mathrm{T}}(10)$.

\section{DISCUSSION}

Because neuroinflammation plays a crucial role in ALS, the availability of PET ligands that can visualize and quantify this process would be useful to guide patient selection in clinical trials and to allow in vivo therapeutic monitoring. In this study, we compared a second-generation TSPO radioligand, ${ }^{18}$ F-DPA714, with a promising novel neuroinflammation radioligand, ${ }^{11} \mathrm{C}$ JNJ717, as a proxy for P2X7R distribution in clinical ALS.

P2X7R involvement in ALS has been demonstrated preclinically in mouse models, in which blocking could be neuroprotective in early disease and neurotoxic in later phases, depending on the time of administration. Such a dual mode for P2X7R has been confirmed in microglia of SOD1-G93A mice, for which P2X7R activation releases both proinflammatory markers and antiinflammatory markers. It was hypothesized that P2X7R becomes important around the switch from the antiinflammatory to the proinflammatory phenotype (13). Also, clinically, increased P2X7R immunoreactivity has been observed in the spinal cord of ALS patients (14).

Our results show a visual increase in ${ }^{18} \mathrm{~F}$-DPA714 binding in the MC in ALS patients on an individual level, whereas no regional increase in ${ }^{11} \mathrm{C}$-JNJ717 binding was observed. Additionally, in the patient with associated frontotemporal dysfunction, an increased ${ }^{18} \mathrm{~F}$-DPA714 uptake in the frontal cortex was observed, concordant with the literature (15). The ${ }^{18} \mathrm{~F}-\mathrm{DPA} 714 \mathrm{~V}_{\mathrm{T}}$ ratio of MC to OC was $13 \%$ higher in ALS patients than in HVs, confirming our visual analysis. This increase was apparent both in vivo and in vitro and colocalized with microglial expression. Possibly, P2X7R might be upregulated in the brain only during early phases of the disease, concordant with preclinical results, as $\mathrm{P} 2 \mathrm{X} 7 \mathrm{R}$ is known to drive microglial activation (16). Moreover, in symptomatic ALS as studied in this report, the upregulation of P2X7R might be limited, necessitating the development of ligands with a greater specific-to-nondisplaceable ratio.

A limitation of the study is the lack of direct target (TSPO and P2X7R) immunohistochemistry. Nevertheless, the specificity of both tracers has been demonstrated in previous research (9,17-19). Future studies including direct target availability across the different disease stages are needed to validate our results and to investigate the presymptomatic and late symptomatic stages.

\section{CONCLUSION}

This study demonstrates that TSPO imaging — despite several drawbacks — outperforms P2X7R imaging in early symptomatic ALS patients.

\section{DISCLOSURE}

Koen Van Laere and Philip Van Damme are senior clinical investigators of the Fund for 
Scientific Research, Flanders, Belgium (FWO). Donatienne Van Weehaeghe $(1179620 \mathrm{~N})$ and Evelien Van Schoor (1S46219N) are PhD fellows of the FWO. Philip Van Damme is supported by the ALS Liga België and the KU Leuven funds "Een Hart voor ALS" and "Laeversfonds voor ALS Onderzoek." Dietmar Thal receives funds from FWO, KU Leuven, and collaborates with GE Healthcare, Janssen Pharmaceuticals, and Novartis Pharma AG. Dietmar Thal received travel reimbursement or honoraria from Novartis Pharma AD, GE Healthcare U.K., and UCB. No other potential conflict of interest relevant to this article was reported.

\section{ACKNOWLEDGMENTS}

We thank Kwinter Porters and Jozef Van Loock, the PET radiopharmacy and medical physics team of UZ.

\section{KEY POINTS}

QUESTION: What is the optimal neuroinflammation target in early symptomatic ALS: TSPO or P2X7R?

PERTINENT FINDINGS: In this cross-sectional study on early symptomatic ALS patients, increased TSPO uptake (13\%) in the $\mathrm{MC}$ without a regional increase in P2X7R was observed.

IMPLICATIONS FOR PATIENT CARE: Visualization of neuroinflammation might be used as a stratification and pharmacodynamic biomarker in therapeutic studies; therefore, optimal target determination across different disease stages is important.

\section{REFERENCES}

1. Boillée S, Yamanaka K, Lobsiger CS, et al. Onset and progression in inherited ALS determined by motor neurons and microglia. Science. 2006;312:13891392 .

2. Butovsky O, Jedrychowski MP, Moore CS, et al. Identification of a unique TGFbeta-dependent molecular and functional signature in microglia. Nat Neurosci. 2014;17:131-143.

3. Sargsyan SA, Monk PN, Shaw PJ. Microglia as potential contributors to motor neuron injury in amyotrophic lateral sclerosis. Glia. 2005;51:241-253.

4. Corcia P, Tauber C, Vercoullie J, et al. Molecular imaging of microglial activation in amyotrophic lateral sclerosis. PLoS One. 2012;7:e52941.
5. Turner MR, Cagnin A, Turkheimer FE, et al. Evidence of widespread cerebral microglial activation in amyotrophic lateral sclerosis: an $\left[{ }^{11} \mathrm{C}\right](\mathrm{R})-\mathrm{PK} 11195$ positron emission tomography study. Neurobiol Dis. 2004;15:601-609.

6. Zürcher NR, Loggia ML, Lawson R, et al. Increased in vivo glial activation in patients with amyotrophic lateral sclerosis: assessed with $\left[{ }^{11} \mathrm{C}\right]-\mathrm{PBR} 28$. Neuroimage Clin. 2015;7:409-414.

7. Cerami C, Iaccarino L, Perani D. Molecular imaging of neuroinflammation in neurodegenerative dementias: the role of in vivo PET imaging. Int J Mol Sci. 2017;18:E933.

8. Owen DR, Howell OW, Tang SP, et al. Two binding sites for $\left[{ }^{3} \mathrm{H}\right] \mathrm{PBR} 28$ in human brain: implications for TSPO PET imaging of neuroinflammation. J Cereb Blood Flow Metab. 2010;30:1608-1618.

9. Ory D, Celen S, Gijsbers R, et al. Preclinical evaluation of a P2X7 receptorselective radiotracer: PET studies in a rat model with local overexpression of the human P2X7 receptor and in nonhuman primates. J Nucl Med. 2016;57:14361441.

10. Van Weehaeghe D, Koole M, Schmidt M, et al. $\left[{ }^{11} \mathrm{C}\right] J N J 54173717$, a novel P2X7 receptor radioligand as marker for neuroinflammation: human biodistribution, dosimetry, brain kinetic modelling and quantification of brain $\mathrm{P} 2 \mathrm{X} 7$ receptors in patients with Parkinson's disease and healthy volunteers. Eur J Nucl Med Mol Imaging. 2019;46:2051-2064.

11. Brettschneider J, Del Tredici K, Toledo JB, et al. Stages of pTDP-43 pathology in amyotrophic lateral sclerosis. Ann Neurol. 2013;74:20-38.

12. Desikan RS, Segonne F, Fischl B, et al. An automated labeling system for subdividing the human cerebral cortex on MRI scans into gyral based regions of interest. Neuroimage. 2006;31:968-980.

13. Cieślak M, Roszek K, Wujak M. Purinergic implication in amyotrophic lateral sclerosis: from pathological mechanisms to therapeutic perspectives. Purinergic Signal. 2019;15:1-15.

14. Yiangou Y, Facer P, Durrenberger P, et al. COX-2, CB2 and P2X7-immunoreactivities are increased in activated microglial cells/macrophages of multiple sclerosis and amyotrophic lateral sclerosis spinal cord. BMC Neurol. 2006;6:12.

15. Zhang J. Mapping neuroinflammation in frontotemporal dementia with molecular PET imaging. J Neuroinflammation. 2015;12:108.

16. Monif M, Burnstock G, Williams DA. Microglia: proliferation and activation driven by the P2X7 receptor. Int J Biochem Cell Biol. 2010;42:17531756 .

17. Lavisse S, Inoue $\mathrm{K}$, Jan $\mathrm{C}$, et al. $\left[{ }^{18} \mathrm{~F}\right] \mathrm{DPA}-714$ PET imaging of translocator protein TSPO (18 kDa) in the normal and excitotoxically-lesioned nonhuman primate brain. Eur J Nucl Med Mol Imaging. 2015;42:478-494.

18. Ory D, Postnov A, Koole M, et al. Quantification of TSPO overexpression in a rat model of local neuroinflammation induced by intracerebral injection of LPS by the use of $\left[{ }^{18}\right.$ F]DPA-714 PET. Eur J Nucl Med Mol Imaging. 2016;43:163-172.

19. Ory D, Planas A, Dresselaers T, et al. PET imaging of TSPO in a rat model of local neuroinflammation induced by intracerebral injection of lipopolysaccharide. Nucl Med Biol. 2015;42:753-761. 